\title{
Abdominal pain: do not forget Thorotrast!
}

\author{
Eric Weber, Fatima Laarbaui, Luc Michel, Julian Donckier
}

\section{Summary}

The use of Thorotrast ( $25 \%$ thorium dioxide), a radiologic contrast agent used up until the mid-1950s, was associated with a wide range of malignancies, mainly of hepatic origin. We report a case of Thorotrast-induced hepatocarcinoma in an 82-year-old woman.

Keywords: Thorotrast, hepatocarcinoma, carcinogen

Thorotrast (25\% thorium dioxide), a radiologic contrast agent used between 1928 and 1955, was abandoned following a report by MacMahon $^{1}$ of hepatic angiosarcoma attributed to Thorotrast exposure. Thorium dioxide has radioactive properties due to the emission of alpha-, beta- and gamma-rays with a biological half-life of 400 years. $^{2}$ Its use has been associated with the development of a wide range of malignancies, mainly of hepatic origin. ${ }^{3}$ We here report an additional case of Thorotrast-induced hepatocarcinoma.

\begin{tabular}{|l|}
\hline Thorotrast \\
\hline Thorium \\
- a radioactive compound $(90 \% \alpha$-rays, $9 \%$ \\
- $\beta$-rays, $1 \% \gamma$-rays $)$ \\
- phological half-life: 400 years \\
Indications \\
- cerebral arteriography and ventriculography \\
- hepato-porto-splenography \\
- instillation for visualising body cavities \\
- pyelography \\
Composition \\
- $25 \%$ colloidal solution of thorium dioxide \\
(ThO 14 million years \\
- $20 \%$ dextran \\
- $0.15 \%$ methyl hydroxybenzoate \\
$\left(=200\right.$ mg of ${ }^{232}$ thorium $\left./ \mathrm{ml}\right)$ \\
\hline
\end{tabular}

University Hospital of Mont-Godinne, B-5530 Yvoir, Belgium Division of Internal

Medicine and Endocrinology E Weber

F Laarbaui

J Donckier

Division of Surgery

L Michel

Accepted 9 December 1994

\section{Case report}

A 82-year-old woman was admitted with sudden right-sided abdominal pain. An aortic valvular replacement had been performed eight years earlier and the patient had experienced three interventions for inguinal hernia correction and ovariectomy in the 1940s. Physical examination revealed abdominal tenderness in the right upper quadrant. The erythrocyte sedimentation rate was $44 \mathrm{~mm} / \mathrm{h}$, fibrinogen $670 \mathrm{mg} / \mathrm{dl}$, bilirubin $2.9 \mathrm{mg} / \mathrm{dl}$, serum aspartate aminotransferase $75 \mathrm{UI} / 1$, serum alanine aminotransferase $29 \mathrm{UI} / \mathrm{l}, \gamma$-glutamyl transferase $54 \mathrm{UI} / 1$, lactic dehydrogenase $678 \mathrm{U} / 1$, and alkaline phosphatase $203 \mathrm{U} / \mathrm{l}$. Serology for viral hepatitis indicated immunity for virus $B$ but not for virus $A$ and $C$. Anti-smooth muscle and anti-mitochondria antibodies were absent. Carcinoembryologic antigen was normal and $\alpha$-foetoprotein slightly elevated to $7.1 \mathrm{ng} / \mathrm{ml}$. Ferritin was normal. A plain film of the abdomen demonstrated fine, irregular metallic densities distributed throughout the liver, spleen and parapancreatic lymph nodes (figure 1). This pattern was pathognomonic for Thorotrast deposits. A computed tomographic (CT) scan without contrast injection demonstrated hyperdensities in the liver, spleen and lymph nodes of the hilar region and disclosed a tumoral lesion in segment IV (figure 2), which was confirmed by magnetic resonance imaging (MRI). The diagnosis of hepatocarcinoma induced by Thorotrast exposure was proved by a liver biopsy showing black pigmented deposits in the vicinity of the tumor. Surprisingly, the patient did not remember such an injection. Given the patient's age, cardiovascular state and the poor prognosis, therapeutic abstention was decided. The initials symptoms resolved spontaneously but the patient died a year after the diagnosis.

\section{Comments}

When injected into the vessels, Thorotrast is deposited in the reticuloendothelial system (liver, spleen, bone marrow, lymph nodes). The association with hepatic, haematologic, pulmonary and urinary malignancies has been clearly described. ${ }^{3-5}$ The mechanism by which tumours are induced involves internal irradiation with chromosomic mutations and abnormalities, but also a possible direct toxicity. ${ }^{6}$ Dahlgreen proposed three criteria that must be met before implicating Thorotrast as a cause of neoplasia. First, Thorotrast must be present in the vicinity of the tumour. Second, the latent 
A

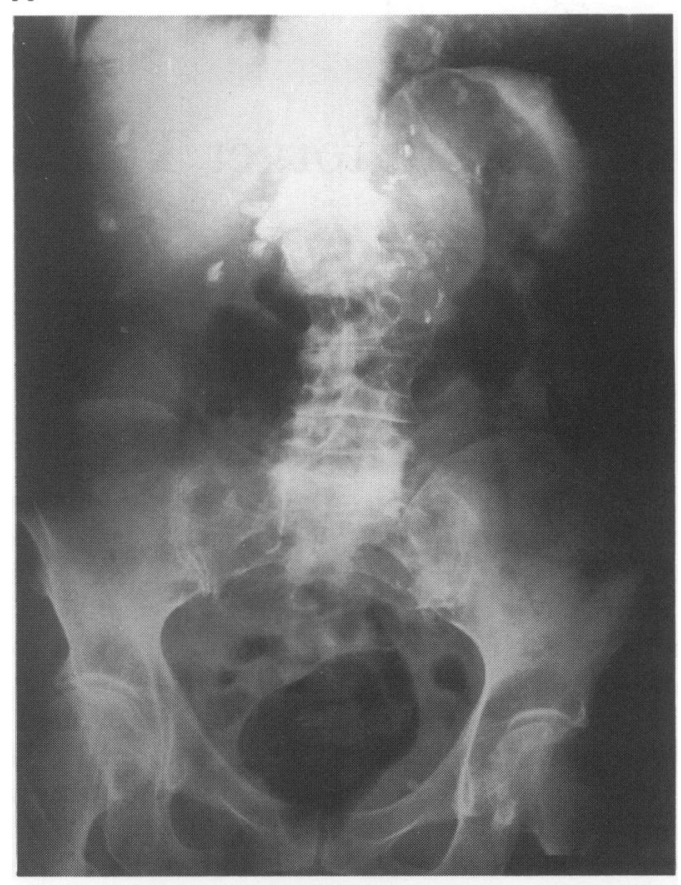

B

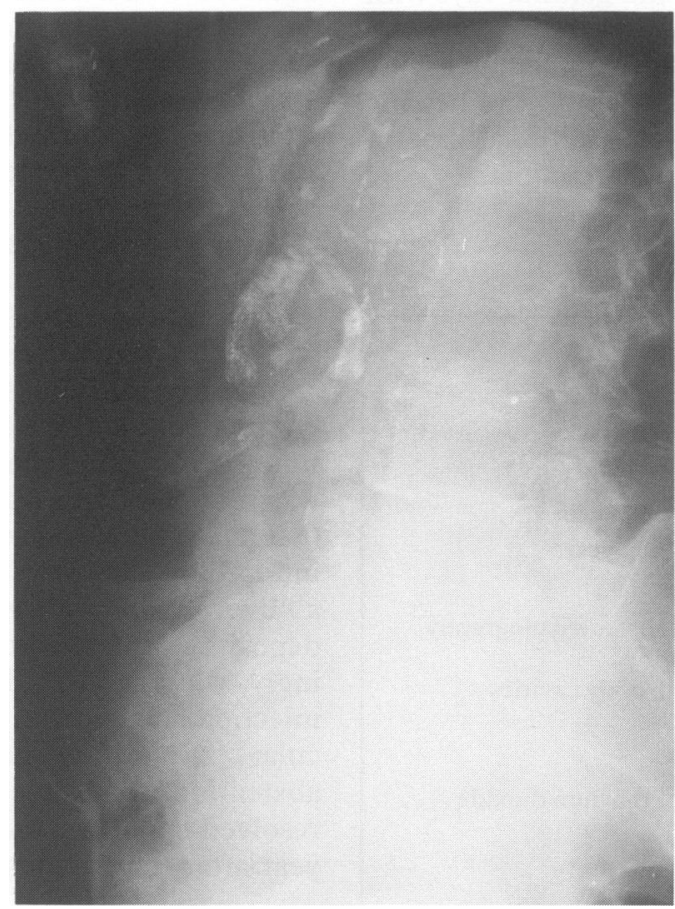

Figure 1 Plain film of the abdomen (anteroposterior (A) and profile (B) incidences). Fine, irregular hyperdensities throughout the liver with a Riedle lobe seen in the right flank. Most important hyperdensities in the lymph nodes of the hilar region of the liver

1 MacMahon $\mathrm{H}$, Murphy A, Bates $\mathbf{M}$. Endothelial cell sarcoma of the liver following Thorotrast (thorium dioxide) administration. $A \mathcal{F} R$ 1947; 83: 163-85.

2 Hursh J, Stedman L, Looney W, et al. Excretion of thorium and thorium daughters after Thorotrast administration to human subjects. Acta Radiol 1957; 47: 481-98.

3 Yuji I, Masamichi K, Toshiro N, Takasaburo M. Pathomorphologic characteristics of 102 cases of Thorotrast-related hepatocellular carcinoma, cholangiocarcinoma and hepatic angiosarcoma. Cancer 1988; 62: 1153-62.

4 Silpananta P, Illescas F, Sheldon H. Multiple malignant neoplasms 40 years after angiography with Thorotrast. Can Med Assoc F 1983; 128: 289-92.

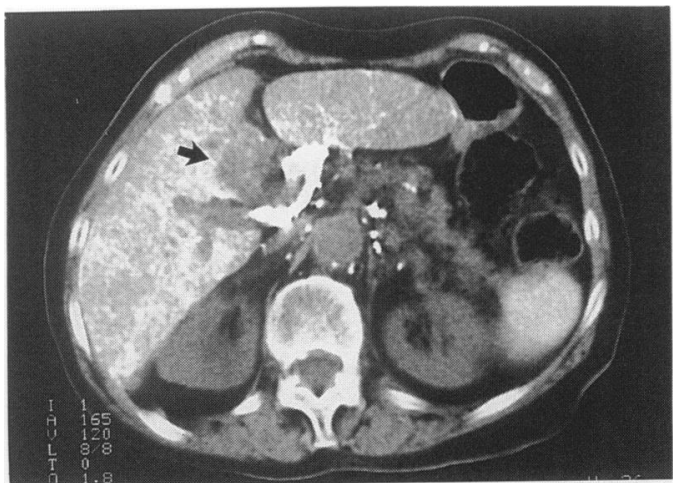

Figure 2 CT without contrast injection of the upper abdomen. Liver, spleen and lymph node hyperdensities are seen spontaneously. A hepatocarcinoma in segment IV of the liver (arrow) is also identified

period must have been sufficiently long (average 20 years). Third, the dose must have been sufficiently high. ${ }^{7}$

As illustrated by the present case, the diagnosis of Thorotrast exposure is made upon the basis of metallic hyperdensities in the liver, spleen and lymph nodes, detected by a plain film of the abdomen which shows the pathognomonic increased density of liver, spleen and lymph nodes. Such a finding should alert the clinician to the possibility of radiation-induced malignancy. CT will confirm this, while MRI and ultrasound do not provide any further information. A liver biopsy will confirm the malignancy while the presence of Thorotrast can be confirmed by autoradiography.

The risk of developing malignancies as a result of Thorotrast exposure continues throughout the patient's life as the radiation is continuous and linked with the long half-life of Thorotrast. In contrast, other carcinogens have usually ceased to give an increased risk at about 30 years after cessation of exposure (15 years in the case of cigarettes).

In conclusion, this case leads us to remind recently trained clinicians of this historical pathology, which obviously will disappear with time but must be kept in mind for at least two decades, when confronted with atypical abdominal pain in elderly patients.

\section{Learning points}

- recall of the carcinogenic role of Thorotrast

- radiologic pathognomonic patterns of Thorotrast deposits

- diagnostic interest of the plain film of the abdomen

- 'in vivo' confirmation of the carcinologic effects of radiation

5 Kessel D, Robbens R, Wilkinson M, Smith P. Thorium dioxide, forgotten but not gone. $\mathrm{Br} \mathcal{F}$ Radiol 1991; 64: 1067-9.

6 Collart F, Horio M, Schlenker R, Kathren R, Huberman E. Alteration of the c-Fms gene in a blood sample from a Thorotrast individual. Health Phys 1992; 63: 27-32.

7 Dahlgreen S. Late effects of thorium dioxide on the liver of patients in Sweden. Ann NY Acad Sci 1967; 145: 718-27. 